\title{
Strong Negation in Well-Founded and Partial Stable Semantics for Logic Programs
}

\author{
Pedro Cabalar ${ }^{1}$, Sergei Odintsov ${ }^{2}$ and David Pearce ${ }^{3 \star}$ \\ 1 Corunna University (Corunna, Spain), cabalar@udc.es \\ 2 Sobolev Institute of Mathematics (Novosibirsk, Russia), odintsov@math.nsc.ru \\ ${ }^{3}$ Universidad Rey Juan Carlos (Madrid, Spain), davidandrew.pearce@urjc.es
}

\begin{abstract}
A formalism called partial equilibrium logic (PEL) has recently been proposed as a logical foundation for the well-founded semantics (WFS) of logic programs. In PEL one defines a class of minimal models, called partial equilibrium models, in a non-classical logic, $H T^{2}$. On logic programs partial equilibrium models coincide with Przymusinski's partial stable (p-stable) models, so that PEL can be seen as a way to extend WFS and p-stable semantics to arbitrary propositional theories. We study several extensions of PEL with strong negation and compare these with previous systems extending WFS with explicit negation, notably WSFX [10] and p-stable models with "classical" negation [11].
\end{abstract}

\section{Introduction}

The well-founded semantics (WFS) of [13] provides one of the most established approaches to logic programming, and the well-known implementation XSBProlog $^{4}$ is extensively used in AI problem solving. Closely related to WFS is the semantics of partial stable models due to Przymusinski [11]. Partial stable (henceforth p-stable) models provide a natural generalisation of stable models [5] to a multi-valued setting and on normal logic programs capture the well-founded model as a special (minimal model) case.

Stable, p-stable and well-founded semantics have all been extended with a second negation operator representing explicit falsity. In the first two cases this was originally called classical negation $[6,11]$, in the latter case explicit negation [10]. Nowadays the terms "strong" and "explicit" are often used interchangeably to denote this second form of negation.

The term strong negation has its roots in logic and refers to the concept of constructible falsity introduced by Nelson [8] and later presented in the form of an axiomatic system by Vorob'ev [14,15]. In the case of stable models or answer sets it is appropriate to label the second negation "strong" because these models correspond in an exact fashion to minimal models in an extension of Nelson's logic, sometimes called $\mathbf{N}_{\mathbf{5}}$ (standing for 5 truth-values). A nonmonotonic extension of $\mathbf{N}_{\mathbf{5}}$, called equilibrium logic, yields a foundation for answer set semantics

\footnotetext{
* This research was partially supported by CICyT project TIC-2003-9001-C02.

${ }^{4}$ See http://www.cs.sunysb.edu/ sbprolog/xsb-page.html
} 
as well as a means to extend the syntax of answer set programs to arbitrary propositional theories $[9,7,4]$. Another, equivalent way to view $\mathbf{N}_{\mathbf{5}}$ is to take the superintuitionistic logic of here-and-there, $(H T)$, and form its least extension by strong negation satisfying the Vorob'ev axioms.

These logical foundations for answer set programs with strong negation have been extensively studied in the literature. Until recently there was no comparable foundational study for well-founded and p-stable semantics; an underlying, monotonic base logic was missing. Recently this situation has changed with the development of partial equilibrium logic (PEL), a nonmonotonic formalism proposed in [18] as a logical foundation for well-founded and p-stable semantics. In PEL one defines a class of minimal models, called partial equilibrium (p-equilibrium) models, inside a non-classical logic $H T^{2}$. It is shown in $[18,19]$ that, on normal and disjunctive logic programs, p-equilibrium models coincide with p-stable models, so that PEL can be seen as a way to extend WFS and p-stable semantics to arbitrary propositional theories.

In this paper we study the result of adding strong negation ' $\sim$ ' to $H T^{2}$ and hence to partial equilibrium logic. It is useful to work with a conservative extension $H T_{u}^{2}$ of $H T^{2}$ formed by adding a constant ' $u$ ' for 'undefined'. We consider a basic logical system $H T_{u}^{2 \sim}$ and two different extensions of it. All three are conservative extensions of $H T_{u}^{2}$. The base system is fully paraconsistent in the sense that a literal and its contrary may be true in a single model. One extension, $H T_{s c}^{2}$, obeys a "semi-consistency" property requiring that a literal and its contrary cannot be true in a single model. Another extension, $H T_{c o h}^{2}$, obeys a form of the so-called coherence principle [10], sometimes formulated by $\sim L \rightarrow \neg L$, for any literal $L$, where ' $\neg$ ' is default negation. Corresponding to these three underlying logics, one obtains different variants of partial equilibrium logic with strong negation.

While $H T_{u}^{2 \sim}$ can already be considered suitable for handling paraconsistent strong negation in this setting, its extensions relate to semantics proposed for explicit negation in logic programming. Specifically we show that PEL based on $H T_{s c}^{2}$ captures precisely Przymusinski's partial stable semantics for programs

with "classical" negation [11]. Furthermore, by a suitable encoding of program rules into a particular kind of implications, the semantics WSFX of Pereira and Alferes [10] is also interpretable in $H T_{s c}^{2}$. Finally, $H T_{c o h}^{2}$ captures the so-called WFS with "strong negation" as defined in [2].

\section{Routley semantics and strong negation}

The logic $H T^{2}$ underlying partial equilibrium logic was defined in [18] as a finitevalued extension of a logic $N^{*}$ whose semantics is given by direct combination of Kripke semantics for intuitionistic logic and Routley style semantics for weak negation $\neg$ [12]. Prior to considering the version of $H T^{2}$ with strong negation, we first introduce this kind of negation to $N^{*}$, leading to a new logic we will denote as $N^{* \sim}$. Formulas of $N^{*}$ are built-up in the usual way using the logical constants: $\wedge, \vee, \rightarrow, \neg$, standing respectively for conjunction, disjunction, implication and 
weak negation; $N^{* \sim}$ adds to these strong negation, $\sim$. The rules of inference for $N^{*}$ and $N^{* \sim}$ are modus ponens and the contraposition rule for weak negation

$$
\text { (RC) } \frac{\alpha \rightarrow \beta}{\neg \beta \rightarrow \neg \alpha}
$$

The axioms of $N^{*}\left(N^{* \sim}\right)$ are as follows:

1. the axiom schemes of positive logic,

2. weak negation axioms:

$$
\begin{aligned}
& \text { W1. } \neg \alpha \wedge \neg \beta \rightarrow \neg(\alpha \vee \beta) \quad \text { W2. } \neg(\alpha \wedge \beta) \rightarrow \neg \alpha \vee \neg \beta \\
& \text { W3. } \neg(\alpha \rightarrow \alpha) \rightarrow \beta
\end{aligned}
$$

3. and for $N^{* \sim}$ one adds the following axiom schemata involving strong negation taken from the calculus of Vorob'ev $[14,15]$ :

$$
\begin{aligned}
& \text { N1. } \sim(\alpha \rightarrow \beta) \leftrightarrow \alpha \wedge \sim \beta \quad \text { N2. } \sim(\alpha \wedge \beta) \leftrightarrow \sim \alpha \vee \sim \beta \\
& \text { N3. } \sim(\alpha \vee \beta) \leftrightarrow \sim \alpha \wedge \sim \beta \quad \text { N4. } \sim \sim \alpha \leftrightarrow \alpha \\
& \text { N5. } \sim \neg \alpha \leftrightarrow \alpha
\end{aligned}
$$

We introduce a notion of frame in the style of Routley [12].

Definition 1 ( $N^{* \sim}$ model). A Routley or $N^{* \sim}$ frame is a triple $\langle W, \leq, *\rangle$, where $W$ is a set, $\leq$ a partial order on $W$ and $*: W \longrightarrow W$ is such that $x \leq y$ iff $y^{*} \leq x^{*}$. An $N^{* \sim}$ model $\mathcal{M}=\left\langle W, \leq, *, V^{+}, V^{-}\right\rangle$is an $N^{* \sim}$ frame $\langle W, \leq, *\rangle$ together with two valuations $V^{+}, V^{-}: A t \times W \longrightarrow\{0,1\}$ satisfying the condition:

$$
V^{+(-)}(p, u)=1 \quad \& \quad u \leq w \Rightarrow V^{+(-)}(p, w)=1
$$

Valuations extend to all formulas in the following way:

- $V^{+}(\varphi \wedge \psi, w)=1$ iff $V^{+}(\varphi, w)=V^{+}(\psi, w)=1$

- $V^{+}(\varphi \vee \psi, w)=1$ iff $V^{+}(\varphi, w)=1$ or $V^{+}(\psi, w)=1$

- $V^{+}(\varphi \rightarrow \psi, w)=1$ iff for every $w^{\prime}$ such that $w \leq w^{\prime}$, $V^{+}\left(\varphi, w^{\prime}\right)=1 \Rightarrow V^{+}\left(\psi, w^{\prime}\right)=1$

- $V^{+}(\neg \varphi, w)=1$ iff $V^{+}\left(\varphi, w^{*}\right)=0$

- $V^{+}(\sim \varphi, w)=1$ iff $V^{-}(\varphi, w)=1$

- $V^{-}(\varphi \wedge \psi, w)=1$ iff $V^{-}(\varphi, w)=1$ or $V^{-}(\psi, w)=1$

- $V^{-}(\varphi \vee \psi, w)=1$ iff $V^{-}(\varphi, w)=V^{-}(\psi, w)=1$

- $V^{-}(\varphi \rightarrow \psi, w)=1$ iff $V^{+}(\varphi, w)=1$ and $V^{-}(\psi, w)=1$

- $V^{-}(\neg \varphi, w)=1$ iff $V^{+}(\varphi, w)=1$

- $V^{-}(\sim \varphi, w)=1$ iff $V^{+}(\varphi, w)=1$

The only difference between $N^{* \sim}$ models and Routley models for the logic $N^{*}$ as defined in [18] is the presence of two valuations $V^{+}$and $V^{-}$and valuation $V^{+}$ is defined in exactly the same way as the only valuation $V$ of a Routley model. We read $V^{+}(\varphi, w)=1$ as " $\varphi$ is verified at $w$ " and $V^{-}(\varphi, w)=1$ as " $\varphi$ is falsified at w". A proposition $\varphi$ is said to be true in an $N^{* \sim}$ model $\mathcal{M}=\left\langle W, \leq, R, V^{+}, V^{-}\right\rangle$, and we write $\mathcal{M}=\varphi$, if $V^{+}(\varphi, v)=1$, for all $v \in W$. A formula $\varphi$ is valid, in 
symbols $=\varphi$, if it is true in every $N^{* \sim}$ model. It is easy to prove by induction that condition (1) above holds for any formula $\varphi$, ie, $V^{+(-)}(\varphi, u)=1 \& u \leq$ $w \Rightarrow V^{+(-)}(\varphi, w)=1$. Moreover $N^{* \sim}$ is complete for this semantics in the sense that a formula is valid iff it is a theorem of $N$. Completeness proof for $N^{* \sim}$ can be obtained via an easy modification of the canonical model method used in [18] to prove the completeness of $N^{*}$ wrt Routley models.

Note that axiom (W3) allows to define an intuitionistic negation ' - ' in $N^{* \sim}$. Fix some propositional variable $p_{0}$ and put $\perp:=\neg\left(p_{0} \rightarrow p_{0}\right)$ and $-\alpha:=\alpha \rightarrow \perp$, then the $\langle\vee, \wedge, \rightarrow,-\rangle$-fragment of $N^{* \sim}$ coincides with intuitionistic logic.

Verification and falsification for $\vee, \wedge, \rightarrow$ and $\sim$ was defined in exactly the same way as for paraconsistent Nelson's logic $N^{-}$[17], at the same time verification conditions for $\vee, \wedge, \rightarrow$ and $\neg$ are the same as validity conditions for these connective in the logic $N^{*}$ [18], whence

Proposition 1. The logic $N^{* \sim}$ is a conservative extension of both $N^{-}$and $N^{*}$.

We say that a formula is in negative normal form ( $n n f)$ if it contains strong negation only in front of atoms. The strong negation axioms allow one to move strong negation through all other connectives, therefore, we have

Proposition 2. For any formula $\varphi$, there is a formula $\psi$ in $\operatorname{nnf}$ such that $N^{* \sim} \vdash$ $\varphi \leftrightarrow \psi$.

Assume that we have chosen one or another procedure for reducing a formula $\varphi$ to its $n n f \bar{\varphi}$. Now we assign to each atom $p \in A t$ the new atom $p^{\prime}$ and to each formula $\varphi$ the formula $\varphi^{\prime}$ obtained by replacing in $\bar{\varphi}$ each subformula $\sim p$ by $p^{\prime}$. In this way we obtain an embedding of $N^{* \sim}$ into $N^{*}$. This method for eliminating strong negation was discovered by Vorob'ev $[14,15]$.

Proposition 3 (Vorob'ev reduction). $N^{* \sim} \vdash \varphi$ iff $N^{*} \vdash \varphi^{\prime}$.

\section{$3 \quad H T^{2}$ with strong negation}

Given a semantic characterisation of some $N^{*}$ extension determined via a class of $N^{*}$ frames, the least extension of this logic with strong negation can be defined by considering $N^{* \sim}$ models over the same class of frames. In this way we obtain the definition of $H T^{2 \sim}$ from the logic $H T^{2}$. We first introduce briefly the latter.

The logical constants of $H T^{2}$ are $\wedge, \vee, \rightarrow, \neg$. Intuitionistic negation '-' is definable as $-\alpha:=\alpha \rightarrow \neg\left(p_{0} \rightarrow p_{0}\right)$. The axioms and inference rules are those of $N^{*}$ together with the schemata:

$$
\begin{array}{ll}
\text { W4. } & -\alpha \vee--\alpha \\
\text { W5. } & -\alpha \vee(\alpha \rightarrow(\beta \vee(\beta \rightarrow(\gamma \vee-\gamma)))) \\
\text { W6. } & \wedge_{i=0}^{2}\left(\left(\alpha_{i} \rightarrow \bigvee_{j \neq i} \alpha_{j}\right) \rightarrow \bigvee_{j \neq i} \alpha_{j}\right) \rightarrow \bigvee_{i=0}^{2} \alpha_{i} \\
\text { W7. } & \alpha \rightarrow \neg \neg \alpha \\
\text { W8. } & \alpha \wedge \neg \alpha \rightarrow \neg \beta \vee \neg \neg \beta \\
\text { W9. } & \neg \alpha \wedge \neg(\alpha \rightarrow \beta) \rightarrow \neg \neg \alpha \\
\text { W10. } & \neg \neg \alpha \vee \neg \neg \beta \vee \neg(\alpha \rightarrow \beta) \vee \neg \neg(\alpha \rightarrow \beta) \\
\text { W11. } & \neg \neg \alpha \wedge \neg \neg \beta \rightarrow(\alpha \rightarrow \beta) \vee(\beta \rightarrow \alpha)
\end{array}
$$


and the rule $(E C) \frac{\alpha \vee(\beta \wedge \neg \beta)}{\alpha}$

$H T^{2}$ and $H T^{2 \sim}$ models are based on the same notion of frame:

Definition 2 ( $H T^{2 \sim}, H T^{2}$ frame). An $H T^{2 \sim}\left(H T^{2}\right)$ frame is an $N^{* \sim}$ frame $\mathcal{M}=\langle W, \leq, *\rangle$ such that (i) $W$ comprises 4 worlds denoted by $h, h^{\prime}, t, t^{\prime}$, (ii) $\leq$ is a partial ordering on $W$ satisfying $h \leq t, h \leq h^{\prime}, h^{\prime} \leq t^{\prime}$ and $t \leq t^{\prime}$, (iii) the *-operation is given by $h^{*}=t^{*}=t^{\prime}, h^{*}=t^{*}=t$.

Definition 3 ( $H T^{2 \sim}, H T^{2}$ model). An $H T^{2 \sim}$ model $\mathcal{M}=\left\langle W, \leq, *, V^{+}, V^{-}\right\rangle$ is an $H T^{2 \sim}$ frame together with $N^{* \sim}$ valuations $V^{+}, V^{-}: A t \times W \longrightarrow\{0,1\}$. An $H T^{2}$ model is like an $H T^{2 \sim}$ model but with a single valuation $V$ corresponding to $V^{+}$and extended in that way to all $N^{*}$ formulas.

In [18] it was shown that $H T^{2}$ is complete for the class of $H T^{2}$ models.

Proposition 4. The Vorob'ev embedding also holds for $H T^{2 \sim}$, that is: $H T^{2 \sim} \vdash \varphi$ iff $H T^{2} \vdash \varphi^{\prime}$.

With the help of the Vorob'ev reduction one can easily pass from the axioms of $H T^{2}$ to obtain those of $H T^{2 \sim}$.

Theorem 1. $H T^{2 \sim}=H T^{2}+\{\mathbf{N 1}, \ldots, N 5\}$.

Proof. $H T^{2}$ axioms are $\sim$-free and only valuation $V^{+}$is involved in checking their validity. Since $V^{+}$coincides with valuation $V$ of $H T^{2}$ models all these axioms hold in $H T^{2 \sim}$-models too. Vorob'ev axioms hold in all $N^{* \sim}$ models, in particular, in $H T^{2 \sim}$. To prove completeness let us take some $\varphi \notin H T^{2 \sim}$. By Proposition $4, \varphi^{\prime} \notin H T^{2}$. Let $\mathcal{M}^{\prime}=\langle W, \leq, *, V\rangle$ be a counter model for $\varphi^{\prime}$. Defining $V^{+}(p, w)=V(p, w)$ and $V^{-}(p, w)=V\left(p^{\prime}, w\right)$ we obtain a countermodel $\mathcal{M}=\left\langle W, \leq, *, V^{+}, V^{-}\right\rangle$for $\varphi$.

Corollary 1. $H T^{2 \sim}$ is the least $N^{* \sim}$-extension with the property that it is a conservative extension of $H T^{2}$.

The logic $H T^{2 \sim}$ is suitable to handle paraconsistent strong negation. Before defining logics suitable for explosive versions of strong negation with or without a coherence principle we have to introduce an additional constant into $H T^{2}$. Let $u$ be a new constant symbol that will stand for "undefinedness."

Definition 4. An $H T_{u}^{2}$-model is an $H T^{2}$ model, where the constant $u$ is interpreted so that $V(u, h)=V(u, t)=0$ and $V\left(u, h^{\prime}\right)=V\left(u, t^{\prime}\right)=1$.

Denote by $H T_{u}^{2}$ the logic determined by the class of $H T_{u}^{2}$-models.

Theorem 2. $H T_{u}^{2}$ is a conservative extension of $H T^{2}$. Moreover, $H T_{u}^{2}=H T^{2}+$ $\{u \leftrightarrow \neg u\}$. 
The proof is based on an easy observation that formula $p \leftrightarrow \neg p$ is true in an $H T^{2}$ model iff $p$ is true at $h^{\prime}$ and $t^{\prime}$ and false at $h$ and $t$. We define the next three conservative extensions of $H T_{u}^{2}$ with strong negation:

$$
\begin{aligned}
H T_{u}^{2 \sim} & :=H T_{u}^{2}+\{\mathbf{N} 1, \ldots, \mathbf{N} 5\}, \\
H T_{s c}^{2} & :=H T_{u}^{2 \sim}+\{p \wedge \sim p \rightarrow u\}, \\
H T_{c o h}^{2} & :=H T_{u}^{2 \sim}+\{p \rightarrow \neg \sim p \vee u, \sim p \rightarrow \neg p \vee u\} .
\end{aligned}
$$

As above, $H T_{u}^{2 \sim}$ is the least conservative extension of $H T_{u}^{2}$ with strong negation. Models of the other logics are characterised as follows.

Proposition 5. Let $\mathcal{M}=\left\langle W, \leq, *, V^{+}, V^{-}\right\rangle$be an $H T_{u}^{2 \sim}$ model.

(sc) $\mathcal{M}=H T_{s c}^{2}$ iff there is no atom $p$ such that $V^{+}(p, t)=V^{-}(p, t)=1$.

(coh) $\mathcal{M}=H T_{\text {coh }}^{2}$ iff for any atom $p$,

$V^{+}(p, t)=1 \Rightarrow V^{-}\left(p, t^{\prime}\right)=0, V^{-}(p, t)=1 \Rightarrow V^{+}\left(p, t^{\prime}\right)=0$.

One can see that in the case of $H T_{s c}^{2}$ a principle of partial or semi-consistency (sc) is satisfied, whereas for $H T_{c o h}^{2}$ the right-hand condition of Item 2 is equivalent to the coherence principle (coh). Although these conditions will be explained in further detail in the next section, it is easy to see that (coh) implies (sc) and so:

Proposition 6. $H T_{c o h}^{2}$ is stronger than $H T_{s c}^{2}$.

We conclude this section by formulating Vorob'ev reductions for these logics.

Proposition 7. Let $\varphi$ be a formula in the language $\{\vee, \wedge, \rightarrow, \neg, \sim, u\}$ and $A t(\varphi)$ denote the set of its atoms.

1. $H T_{u}^{2 \sim} \vdash \varphi$ iff $H T_{u}^{2} \vdash \varphi^{\prime}$

2. $H T_{s c}^{2} \vdash \varphi$ iff $H T_{u}^{2} \vdash\left(\bigwedge_{p \in A t(\varphi)} p \wedge p^{\prime} \rightarrow u\right) \rightarrow \varphi^{\prime}$.

3. $H T_{\text {coh }}^{2} \vdash \varphi$ iff $H T_{u}^{2} \vdash\left(\bigwedge_{p \in A t(\varphi)}\left(p \rightarrow \neg p^{\prime} \vee u\right) \wedge\left(p^{\prime} \rightarrow \neg p \vee u\right)\right) \rightarrow \varphi^{\prime}$.

\section{Partial equilibrium logic with strong negation}

At each world in an $H T^{2 \sim}$ or $H T_{u}^{2 \sim}$ model $\mathcal{M}=\left\langle W, \leq{ }^{*}, V^{+}, V^{-}\right\rangle$a certain set of literals (atoms or strongly negated atoms) is verified. Let us denote by $H, H^{\prime}, T, T^{\prime}$ the four sets of literals respectively verified at each corresponding world $h, h^{\prime}, t, t^{\prime}$. In case $\mathcal{M}$ is an $H T^{2}$ model, $H, H^{\prime}, T, T^{\prime}$ are simply sets of atoms. More succinctly, we can represent $\mathcal{M}$ as the pair $\langle\mathbf{H}, \mathbf{T}\rangle$ so that we group each pair of unprimed/primed worlds as $\mathbf{H}=\left(H, H^{\prime}\right)$ and $\mathbf{T}=\left(T, T^{\prime}\right)$. Notice that $H \subseteq H^{\prime}$ and $T \subseteq T^{\prime}$ by construction of $\mathcal{M}$. Given such pairs $\mathbf{H}, \mathbf{T}$ we define a partial ordering relation by $\mathbf{H} \leq \mathbf{T}$ iff $H \subseteq T$ and $H^{\prime} \subseteq T^{\prime}$. Note that if $\langle\mathbf{H}, \mathbf{T}\rangle$ is a model then necessarily $\mathbf{H} \leq \mathbf{T}$. The ordering $\leq$ is extended to a partial ordering $\unlhd$ among models as follows. We set $\left\langle\mathbf{H}_{1}, \mathbf{T}_{1}\right\rangle \unlhd\left\langle\mathbf{H}_{2}, \mathbf{T}_{2}\right\rangle$ if (i) $\mathbf{T}_{1}=\mathbf{T}_{2}$; (ii) $\mathbf{H}_{1} \leq \mathbf{H}_{2}$. A model $\langle\mathbf{H}, \mathbf{T}\rangle$ in which $\mathbf{H}=\mathbf{T}$ is said to be total. Note that the term total model does not refer to the absence of undefined literals. To 
represent this, we further say that a total partial equilibrium model is complete if $\mathbf{T}$ has the form $(T, T)$.

Let $\Pi$ be a set of formulas and let $\mathcal{M}$ range over $H T^{2}$ or $H T_{u}^{2 \sim}$ models as appropriate.

Definition 5 (Partial equilibrium model). A model $\mathcal{M}$ of $\Pi$ is said to be a partial equilibrium (or p-equilibrium) model of $\Pi$ if (i) $\mathcal{M}$ is total; (ii) $\mathcal{M}$ is minimal among models of $\Pi$ under the ordering $\unlhd$.

In other words a p-equilibrium model of $\Pi$ has the form $\langle\mathbf{T}, \mathbf{T}\rangle$ and is such that if $\langle\mathbf{H}, \mathbf{T}\rangle$ is any model of $\Pi$ with $\mathbf{H} \leq \mathbf{T}$, then $\mathbf{H}=\mathbf{T}$. Partial equilibrium logic (PEL) is the logic determined by truth in all p-equilibrium models of a theory.

We turn to the relation between PEL and logic programs. A disjunctive (resp. normal) logic program is a set of formulas (also called rules) of the form

$$
a_{1} \wedge \ldots \wedge a_{m} \wedge \neg b_{1} \wedge \ldots \wedge \neg b_{n} \rightarrow c_{1} \vee \ldots \vee c_{k}
$$

where $m, n, k \geq 0$ (resp. $m, n, \geq 0, k=1$ ), and $a, b, c$ with subscripts range over atoms. A program is called extended if $a, b, c$ with subscripts range over objective literals (ie, an atom $p$ or its strong negation $\sim p$ ). For reasons of space we do not repeat here the definition of partial stable (p-stable) models of [11]. A central result of $[18,19]$ is:

Theorem 3 ([19]). A total $H T^{2}$ model $\langle\mathbf{T}, \mathbf{T}\rangle$ is a p-equilibrium model of a disjunctive program $\Pi$ iff $\mathbf{T}$ is a p-stable model of $\Pi$.

The obvious corresponding variants of PEL with strong negation are obtained by considering $H T_{u}^{2 \sim}, H T_{s c}^{2 \sim}$ or $H T_{c o h}^{2 \sim}$ models respectively. Let us now reconsider the conditions given in Proposition 5. We see that models of $H T_{s c}^{2 \sim}$ are 'semi'consistent in that the sets $H, T$ (ie, the "founded" information) do not contain any contrary pairs of literals $p, \sim p$. On the other hand, models of $H T_{c o h}^{2 \sim}$ have the property that $p \in T \Rightarrow \sim p \notin T^{\prime}$ and $\sim p \in T \Rightarrow p \notin T^{\prime}$. Hence in any p-equilibrium model $\mathcal{M}$ over $H T_{\text {coh }}^{2 \sim}$, we have that $\mathcal{M} \models p \Rightarrow \mathcal{M} \models \neg \sim p$ and $\mathcal{M} \models \sim p \Rightarrow \mathcal{M} \models \neg p$. This is exactly the coherence principle formulated in [2].

The fact that the logics $H T_{u}^{2 \sim}, H T_{s c}^{2 \sim}$ and $H T_{c o h}^{2 \sim}$ are reducible to $H T_{u}^{2}$ via the Vorob'ev transformations given in Proposition 7 is not only significant for proving completeness theorems. The transformation from $\varphi$ to $\varphi^{\prime}$ is linear and allows us to transfer some key properties from ordinary PEL to the strong negation variants. This applies in particular to the strong equivalence theorem and complexity results. We say that two sets of formulas $\Gamma_{1}$ and $\Gamma_{2}$ are strongly equivalent, if for any set of formulas $\Gamma, \Gamma_{1} \cup \Gamma$ and $\Gamma_{2} \cup \Gamma$ have the same pequilibrium models.

Theorem 4 ([18]). Sets of $N^{*}$ formulas $\Gamma_{1}$ and $\Gamma_{2}$ are strongly equivalent iff $\Gamma_{1}$ and $\Gamma_{2}$ are equivalent as $H T_{u}^{2}$ theories.

Corollary 2. Theorem 4 continues to hold for sets of $N^{* \sim}$ formulas where strong equivalence is defined wrt any of the given strong negation extensions of PEL and $H T_{u}^{2}$ is replaced by $H T_{u}^{2 \sim}, H T_{s c}^{2 \sim}$ and $H T_{c o h}^{2 \sim}$ as appropriate. 
Proposition 8. The complexity of reasoning tasks in (any of the variants of) PEL with strong negation lies in the same class as that of ordinary PEL. In particular, from [19] it follows that the decision problem for entailment in PEL with strong negation (checking truth in all p-equilibrium models) is $\Pi_{2}^{P}$-hard.

Note that as in the case of answer set semantics, the Vorob'ev transformation allows reducing all strong negation variants to ordinary PEL, whose implementation strategies are discussed elsewhere [19].

\section{Capturing WFS variants with a second negation}

Let us focus now on extended logic programs without disjunction, consisting of rules like (2) where $k=1$, and where subscripted $a, b, c$ are objective literals. We denote $B^{+}(r):=\left\{a_{1}, \ldots, a_{m}\right\}, B^{-}(r):=\left\{b_{1}, \ldots, b_{m}\right\}$ and $H d(r):=c_{1}$. Sometimes $B^{+}(r)$ (resp. $\left.B^{-}(r)\right)$ will be used as the conjunction (resp. the disjunction) of their atoms. The whole body is denoted as $B(r):=B^{+}(r) \wedge \neg B^{-}(r)$. A program is said to be definite if $B^{-}(r)=\emptyset$ for all its rules. Given a set of objective literals $I$ and a definite rule $r$, we write $I \models r$ when $H d(r) \in I$ if $B^{+}(r) \subseteq I$ and $I \models P$ when $I \models r$ for all $r \in P$. The set $I$ is consistent when it does not contain a pair of literals $p$ and $\sim p$. The reduct [5] $P^{I}$ of a normal logic program $P$ wrt interpretation $I$ corresponds to: (i) removing all $r \in P$ with $B^{-}(r) \cap I \neq \emptyset$; and (ii) removing the default literals from the remaining rules. As $P^{I}$ is definite, it has a least model usually represented as $\Gamma_{P}(I)$, or just $\Gamma(I)$ when there is no ambiguity. The seminormal version $P_{s}$ of a program $P$ consists of a rule like $\neg \sim H d(r) \wedge B(r) \rightarrow H d(r)$ per each rule $r \in P$. We write $\Gamma_{s}$ to stand for $\Gamma_{P_{s}}$, when there is no ambiguity about $P$. Furthermore, we consider that $\Gamma_{s}(I)$ is not defined for an inconsistent $I$.

A partial stable (p-stable) model is a pair $I \subseteq J$ satisfying $J=\Gamma(I)$ and $I=\Gamma(J)$. When $I$ is consistent, it is called p-stable model with "classical negation" [11]. When $(I, J)$ further satisfies coherence $(L \in I$ implies $\sim L \notin J)$ it is called p-stable model with "strong negation" [2]. A WFSX p-stable model is any pair of sets of literals $(I, J)$, with $I$ consistent and $I \subseteq J$, satisfying $J=\Gamma_{s}(I)$ and $I=\Gamma(J)$. The well-founded model (WFM) is the least-information p-stable model (if one exists) in each of these variants. The WFM is computable by iteration on operator $\Gamma \Gamma$ or, in the case of WFSX, on $\Gamma \Gamma_{s}$.

Theorem 5. $\langle\mathbf{T}, \mathbf{T}\rangle$ is an $H T_{s c}^{2}$ p-equilibrium model of an extended program $P$ iff $\mathbf{T}$ is a classical-negation p-stable model of $P$.

Theorem 6. $\langle\mathbf{T}, \mathbf{T}\rangle$ is an $H T_{c o h}^{2}$ p-equilibrium model of an extended program $P$ iff $\mathbf{T}$ is a strong-negation p-stable model of $P$.

Our WFSX encoding translates a program $P$ into $P^{\prime}$ adding, for each $r \in P$, the pair of implications: $u \wedge \neg \sim H d(r) \wedge B(r) \rightarrow H d(r)$ and $B(r) \rightarrow H d(r) \vee u$.

Lemma 1. Let $\mathcal{M}=\langle\mathbf{H}, \mathbf{T}\rangle$, with $\mathbf{H}=\left(H, H^{\prime}\right)$ and $\mathbf{T}=\left(T, T^{\prime}\right)$, and let $P$ be an extended logic program. Then $\mathcal{M} \models P^{\prime}$ in $H T_{u}^{2}$ is equivalent to the four conditions: $H^{\prime} \models P_{s}^{T}, H \models P^{T^{\prime}}, T^{\prime} \models P_{s}^{T}$ and $T \models P^{T^{\prime}}$. 
Theorem 7. A pair $\mathbf{T}=\left(T, T^{\prime}\right)$ is a WFSX p-stable model of an extended logic program $P$ iff $\langle\mathbf{T}, \mathbf{T}\rangle$ is an $H T_{s c}^{2}$ p-equilibrium model of $P^{\prime}$.

Proof (sketch). The left to right direction follows quite directly from Lemma 1. For right to left, if $\langle\mathbf{T}, \mathbf{T}\rangle$ is an $H T_{s c}^{2}$ p-equilibrium model of $P^{\prime}$, from Lemma 1 we get $T \models P^{T^{\prime}}$ and $T^{\prime} \mid=P_{s}^{T}$, so we only have to prove their minimality. If we have some $H \subset T, H \models P^{T^{\prime}}$, then we can use Lemma 1 to conclude that the interpretation $\left\langle\left(H, T^{\prime}\right), \mathbf{T}\right\rangle$ would be model of $P^{\prime}$ smaller than $\langle\mathbf{T}, \mathbf{T}\rangle$. If we have some $H^{\prime} \subset T^{\prime}, H^{\prime}=P_{s}^{T}$, something similar happens applying Lemma 1 on the interpretation $\left\langle\left(H^{\prime} \cap T, H^{\prime}\right), \mathbf{T}\right\rangle$, although in this case, to prove that $H^{\prime} \cap T \models P^{T^{\prime}}$ we must use consistency of $T$.

\section{Concluding remarks}

Partial equilibrium logic (PEL) provides a purely logical characterisation of pstable models and hence, as argued in $[18,19]$, yields a natural logical foundation for p-stable semantics and WFS. We have shown here how strong negation can be added to PEL while maintaining the same complexity of reasoning and preserving strong equivalence theorems. While the coherence principle $(\sim L \rightarrow \neg L)$, much discussed in [3], seems plausible for explosive strong negation, originally [10] strong negation was added to logic programs without this principle. When we deal with strong negation in a paraconsistent setting it seems reasonable to explore different options: logics including coherence as in [1] as well as logics without, as defined here. Indeed, if we do not assume that models are consistent, the fact $\sim p$ should not imply that we can not prove $p$. In this way we are led to the three conservative extensions of $H T^{2}$ with strong negation, viz. $H T_{u}^{2 \sim}$, $H T_{s c}^{2 \sim}$ and $H T_{c o h}^{2 \sim}$, where only the latter satisfies coherence.

We saw that PEL over $H T_{c o h}^{2 \sim}$ provides an alternative to the semantics WFSX since its p-equilibrium models do not coincide with the p-stable models of [3]. Evidently there are different solutions for adding a second, coherent negation to programs under WFS. There may be a trade-off between obtaining intuitively correct inferences and computationally desirable properties. For example, WSFX over normal programs enjoys computationally useful properties like relevance [3] (not obeyed by PEL in general), while some example programs discussed above seem to lead to less intuitive inferences under WSFX than under PEL. Nevertheless we have seen a precise sense in which WSFX is interpretable in PEL over $H T_{s c}^{2 \sim}$; moreover PEL offers a genuinely logical treatment of strong negation, defined over arbitrary formulas. In the case of the p-stable semantics of Przymusinski [11], on the other hand, we can derive precise agreement with the version of PEL defined over the logic $H T_{s c}^{2 \sim}$, and therefore obtain a means to extend p-stable semantics to arbitrary propositional theories.

Summarising, we have studied extensions of PEL with strong negation based on natural, logical principles. Capturing WSFX or other specific semantics was not a primary aim. We have provided precise logical characterisations for various extensions using axiomatic systems and we proved Strong Equivalence theorems. As a by-product we showed that some of our extensions are closely related to 
previously proposed semantics. In [1] there is a frame-based approach to WSFX and its paraconsistent variant, WSFXp, but the aims and approach seem to be different. [1] does not discuss logic, provide axiomatisations or strong equivalence theorems. For these reasons, and for reasons of space, we have not provided a comparison with the work of [1]; this topic is postponed for the future.

\section{References}

1. J. Alcântara, C. Damásio \& L. M. Pereira. A Frame-based Characterisation of the Paraconsistent Well-founded Semantics with Explicit Negation. Unpublished draft, available at http://centria.di.fct.unl.pt/ jfla/publications/

2. Alferes, J.J., Pereira, L.M.: On logic programs semantics with two kinds of negation. In: Proc. of the 5th Intl. Joint Conf. and Symposium on Logic Programming, MIT Press (1992) 574-588.

3. J.J. Alferes \& L.M. Pereira. Reasoning with Logic Programming, Springer, 1996.

4. P. Cabalar, D. Pearce \& A. Valverde. Reducing propositional Theories in Equilibrium Logic to Logic Programs in Proc. EPIA 2005, LNAI 3803, Springer, 2005.

5. M. Gelfond and V. Lifschitz. The stable model semantics for logic programming. In Proc. of ICLP'88, pp. 1070-1080, 1988. The MIT Press.

6. M. Gelfond and V. Lifschitz. Classical negation in logic programs and disjunctive databases. New Generation Computing, 9:365-385, 1991.

7. V. Lifschitz, D. Pearce, and A. Valverde. Strongly equivalent logic programs. ACM Transactions on Computational Logic, 2(4):526-541, October 2001.

8. D. Nelson. Constructible falsity. Journal of Symbolic Logic, 14(2):16-26, 1949.

9. D. Pearce. A new logical characterisation of stable models and answer sets. In Proc. of NMELP 96, LNCS 1216, pp. 57-70. Springer, 1997.

10. L. M. Pereira \& J. J. Alferes, Well Founded Semantics for Logic Programs with Explicit Negation, in: European Conference on Artificial Intelligence, B. Neumann (ed.), John Wiley \& Sons 1992, pp.102-106.

11. Przymusinski, T. Stable semantics for disjunctive programs. New Generation Computing 9 (1991), 401-424.

12. R. Routley and V. Routley. The Semantics of First Degree Entailment. Nôus, 6, 335-359, 1972.

13. A. van Gelder, K.A. Ross, and J.S. Schlipf. Unfounded sets and well-founded semantics for general logic programs. JACM, 38(3):620-650, 1991.

14. N. N. Vorob'ev. A constructive propositional calculus with strong negation (in Russian). Doklady Akademii Nauk SSR, 85:465-468, 1952.

15. N. N. Vorob'ev. The problem of deducibility in constructive propositional calculus with strong negation (in Russian). Doklady Akademii Nauk SSR, 85:689-692, 1952.

16. N. N. Vorob'ev. Constructive propositional calculus with strong negation (in Russian). Transactions of Steklov's Institute, 72: 195-227, 1964.

17. H. Wansing. Negation. In: L.Goble(ed.), The Blackwell Guide to Philosophical Logic, Basil Blackwell Publishers, Cambridge/MA, 415-436, 2001.

18. Logical Foundations of Well-Founded Semantics. to appear, 2006.

19. Analysing and Extending Well-Founded and Partial Stable Semantics using Partial Equilibrium Logic. Technical Report, Feb. 2006, submitted for publication. 